\title{
Loss of EMP2 Inhibits Melanogenesis of MNT1 Melanoma Cells via Regulation of TRP-2
}

\author{
Enkhmend Enkhtaivan ${ }^{1, \dagger}$, Hyun Ji Kim ${ }^{1, \dagger}$, Boram Kim¹, Hyung Jung Byun ${ }^{1}$, Lu Yu ${ }^{1}$, Tuan Minh Nguyen ${ }^{1}$, \\ Thi Ha Nguyen ${ }^{1}$, Phuong Anh Do', Eun Ji Kim², Kyung Sung Kim¹, Hiệu Phùng Huy ${ }^{1}$, Mostafizur Rahman ${ }^{1}$, \\ Ji Yun Jang ${ }^{1,3}$, Seung Bae Rho ${ }^{3}$, Ho Lee ${ }^{3}$, Gyeoung Jin Kang ${ }^{2}$, Mi Kyung Park ${ }^{3}$, Nan-Hyung Kim ${ }^{4}$, \\ Chang Ick Choi ${ }^{1}$, Kyeong Lee ${ }^{1}$, Hyo Kyung Han ${ }^{1}$, Jungsook Cho ${ }^{1}$, Ai Young Lee ${ }^{4}$ and Chang Hoon Lee ${ }^{1, *}$ \\ ${ }^{1}$ BK21 FOUR Team and Integrated Research Institute for Drug Development, College of Pharmacy, Dongguk University, Seoul \\ 04620, Republic of Korea \\ ${ }^{2}$ Lillehei Heart Institute, University of Minnesota, Minneapolis, MN 55455, USA \\ ${ }^{3}$ National Cancer Center, Goyang 10408, \\ ${ }^{4}$ Department of Dermatology, Dongguk University Ilsan Hospital, Goyang 10326, Republic of Korea
}

\begin{abstract}
Melanogenesis is the production of melanin from tyrosine by a series of enzyme-catalyzed reactions, in which tyrosinase and DOPA oxidase play key roles. The melanin content in the skin determines skin pigmentation. Abnormalities in skin pigmentation lead to various skin pigmentation disorders. Recent research has shown that the expression of EMP2 is much lower in melanoma than in normal melanocytes, but its role in melanogenesis has not yet been elucidated. Therefore, we investigated the role of EMP2 in the melanogenesis of MNT1 human melanoma cells. We examined TRP-1, TRP-2, and TYR expression levels during melanogenesis in MNT1 melanoma cells by gene silencing of EMP2. Western blot and RT-PCR results confirmed that the expression levels of TYR and TRP-2 were decreased when EMP2 expression was knocked down by EMP2 siRNA in MNT1 cells, and these changes were reversed when EMP2 was overexpressed. We verified the EMP2 gene was knocked out of the cell line (EMP2 CRISPR/Cas9) by using a CRISPR/Cas9 system and found that the expression levels of TRP-2 and TYR were significantly lower in the EMP2 CRISPR/Cas9 cell lines. Loss of EMP2 also reduced migration and invasion of MNT1 melanoma cells. In addition, the melanosome transfer from the melanocytes to keratinocytes in the EMP2 KO cells cocultured with keratinocytes was reduced compared to the cells in the control coculture group. In conclusion, these results suggest that EMP2 is involved in melanogenesis via the regulation of TRP-2 expression.
\end{abstract}

Key Words: EMP2, TYR, TRP-1, TRP-2, Melanogenesis, Melanosome transfer

\section{INTRODUCTION}

Skin pigmentation depends on the cooperation of melanocytes and keratinocytes (KC), and the delivery and distribution of melanosomes to $\mathrm{KC}$ is an important process in skin pigmentation (Lee, 2015; Serre et al., 2018). Skin pigmentation disorders can be widespread, and the resulting pigmentation changes are classified as depigmentation, hypopigmentation, and hyperpigmentation (Enkhtaivan and Lee, 2021). Skin hyperpigmentation is an especially common skin condition and includes melasma, melanoma, and post-inflammatory hyper- pigmentation (Lambert et al., 2019). It is caused by sun exposure, hormones, skin damage, and inflammation. However, the lack of effective therapeutics available to treat skin hyperpigmentation suggests the need for further analysis of appropriate targets and various mechanisms regulating the fate of melanin, including melanogenesis and degradation.

In mammals, melanocytes regulate skin pigmentation by catalyzing melanin biosynthesis via three melanocyte-specific enzymes: tyrosinase (TYR), tyrosinase-related protein 1 (TRP1) and tyrosine-related protein 2 (TRP-2) (Kim et al., 2014; Tuerxuntayi et al., 2014; Ito and Sato, 2021). TYR is involved

\section{Open Access https://doi.org/10.4062/biomolther.2022.001}

This is an Open Access article distributed under the terms of the Creative Commons Attribution Non-Commercial License (http://creativecommons.org/licenses/by-nc/4.0/) which permits unrestricted non-commercial use, distribution, and reproduction in any medium, provided the original work is properly cited.
Received Jan 3, 2022 Revised Feb 7, 2022 Accepted Feb 7, 2022

Published Online Mar 1, 2022

\section{*Corresponding Author}

E-mail: uatheone@dongguk.edu

Tel: +82-31-961-5213, Fax: +82-31-961-5206

${ }^{\dagger}$ The first two authors contributed equally to this work. 
in melanin biosynthesis as a rate-limiting enzyme, catalyzing the hydroxylation of L-tyrosine to L-3, 4-dihydroxyphenylalanine (DOPA) and the oxidation of DOPA to dopaquinone is catalyzed by TYR. Once dopaquinone is oxidized to dopachrome, TRP-2 catalyzes dopachrome to 5,6-dihydroxyindole2-carboxylic acid (Yokoyama et al., 1994; Lee et al., 2011; Enkhtaivan and Lee, 2021). In addition, TRP-1 is involved in melanogenesis by regulating the activity of TYR (Kobayashi et al., 1994).

EMP2 is a member of the growth arrest-specific-3/peripheral myelin protein-22 (GAS3/PMP22) family of tetraspan proteins (Wadehra et al., 2002). It plays a role in the regulation of various processes, including cell migration, cell proliferation, cell contraction, cell adhesion, apoptosis, endocytosis, and cholesterol homeostasis (Wadehra et al., 2008; Wang et al., 2017). EMP2 regulates the surface expression of $\alpha 5 \beta 3$ integrin in the endometrium and regulates adhesion by interacting with $\beta 1$ integrin (Wadehra et al., 2002; Wang et al., 2017). EMP2 is also involved in the regulation of the epithelial-mesenchymal transition (EMT) process and the differentiation of cells into functional cardiomyocytes (Liu et al., 2019). Recent studies have shown that EMP2 is downregulated in melanoma at the protein and mRNA levels because of autophagic proteolysis mediated by the mTOR pathway (Wang et al., 2019).

However, there are no detailed studies of the link between EMP2 and melanogenesis as yet. In this study, we investigated the involvement of EMP2 in melanogenesis. We found that EMP2 regulates the melanogenesis of MNT1 melanoma cells via the control of TRP-2 expression.

\section{MATERIALS AND METHODS}

\section{Materials}

Minimum essential medium (MEM) was purchased from Gibco (Carlsbad, CA, USA). Lipofectamine ${ }^{\mathrm{TM}} 3000$ reagent was purchased from Invitrogen (Carlsbad, CA, USA). AntiEMP2 antibody and anti-tyrosinase antibody were obtained from Abcam (Cambridge, UK). Anti-TRP-1, anti-TRP-2, antiMITF and anti- $\beta$ actin antibodies were purchased from Santa Cruz Biotechnology (Santa Cruz, CA, USA). Alexa FluorTM 488 donkey goat anti-mouse IgG antibody and Alexa Fluor $594^{\circledR}$ goat anti-rabbit IgG antibody were supplied from Molecular Probes (Eugene, OR, USA). AccuPower ${ }^{\circledR}$ PCR PreMix was purchased from Bioneer Inc (Daegu, Korea).

\section{Cell culture}

The human melanoma (MNT-1) cells were acquired as a gift from professor Ai-Young Lee (Department of Dermatology, Dongguk University Ilsan Hospital, Goyang, Korea). MNT-1 cells were maintained in MEM (Gibco) containing 10\% Dulbecco's MEM (Cytiva), 2\% 1M HEPES (Sigma-Aldrich Co., St. Louis, MO, USA), 20\% FBS (Hyclone, South Logan, UT, USA), streptomycin $(100 \mu \mathrm{g} / \mathrm{mL})$, and penicillin $(100 \mathrm{U} / \mathrm{mL})$. The cells were washed twice in Dulbecco's phosphate-buffered saline (DPBS). The cells were maintained at $37^{\circ} \mathrm{C}$ in a humidified atmosphere containing $5 \%$ carbon dioxide.

\section{Gene correlation analysis using GEPIA 2}

The correlation of EMP2 expression levels with TRP-1, TRP-2 or TYR expression in GTEx skin datasets was investigated using Pearson correlation statistics on the Correlation
Analysis module of GEPIA 2 (GTEx Consortium, 2017; Tang et al., 2019).

\section{Western blot}

Western blot analysis was conducted in accordance with a previous report (Rho et al., 2021). Cells were washed twice with PBS and whole-cell lysates were prepared in RIPA buffer containing protease inhibitor cocktail and Xpert Phosphatase Inhibitor Cocktail Solution (GenDEPOT, Barker, TX, USA) on ice for $15 \mathrm{~min}$. Cell lysates were centrifuged at 15,000 rpm for $15 \mathrm{~min}$ at $4^{\circ} \mathrm{C}$, and the resultant supernatants were subjected to Western blotting (Rho et al., 2021). The total protein concentration was quantified using Coomassie brilliant blue. Equal amounts of protein were separated by electrophoresis using a $10 \%$ SDS-polyacrylamide gel and transferred onto a polyvinylidene difluoride (PVDF) membrane. After blocking with $3 \%$ skim milk in TBST for $1 \mathrm{~h}$ at room temperature, the membrane was then incubated with the appropriate primary antibodies in $3 \%$ BSA at $4^{\circ} \mathrm{C}$ overnight. And, after Tris-buffered saline containing Tween (TBST) washing, the membrane was incubated with HRP-conjugated secondary antibody $(1: 5,000)$ at room temperature for $60 \mathrm{~min}$. The proteins were detected using PowerOptiECL detection reagent (Bionote, Seoul, Korea) and exposure to X-ray film.

\section{Reverse transcriptase-polymerase chain reaction (RT-PCR)}

RT-PCR was carried out following the method described in a previous report (Kim et al., 2021a). Total RNA was isolated from cells using the Trizol reagent (Invitrogen). NanoDropTM 2000 was used to measure RNA concentrations and RNA purity. RNA was reverse transcribed into complementary DNA, which was produced from total RNA with oligo(dt) 15 primer (5 $\mu \mathrm{g} / \mu \mathrm{L}), 10 \mathrm{mM} \mathrm{dNTP} \operatorname{mix}(0.5 \mu \mathrm{M}), 5 \times$ reaction buffer, $25 \mathrm{mM}$ $\mathrm{MgCl}_{2}$ and reverse transcriptase. The sequences of PCR primers were: forward EMP2 5'-GCATCGCCTTCTTCATCTTC -3'; reverse EMP2 5'-CCTTCTCTGGTCACGGGATA-3'; forward TRP-1 5'-ACTTTGTAACAGCACCGAGGAT-3', reverse TRP-1 5'-ATGTCCAATAGGGGCATTTTC-3'; forward TRP-2 5'-GATCACCACACAACACTGGC -3', reverse TRP-2 5'-AGAGTCGGATCGTCTGGTCT -3'; forward TYR 5'- TTGG-CAGATTGTCTGTAGCC-3', reverse TYR 5'-AGGCATTGTGCATGGTGCTT -3'; forward GAPDH 5'-TGGTCTCCTCTGACTTCA-3', reverse GAPDH 5'-CGTTGTCATACCAGGAAATG-3'. The PCR profile was $94^{\circ} \mathrm{C}$ for $30 \mathrm{~s}$ (denaturing), $56^{\circ} \mathrm{C}$ for $30 \mathrm{~s}$ (annealing) and $72^{\circ} \mathrm{C}$ for $30 \mathrm{~s}$ (elongation) for 30 cycles. The PCR products were electrophoresed on a $1.5 \%$ agarose gel and photographed under ultraviolet light.

\section{Confocal microscopy}

Confocal microscopy was conducted in accordance with a previous report (Kim et al., 2021b). The MNT1 cells were cultured on coverslips. The cells were fixed in $4 \%(\mathrm{w} / \mathrm{v})$ paraformaldehyde (PFA) for $10 \mathrm{~min}$ at room temperature. The fixed cells were followed by three washes in phosphate-buffered saline (PBS) with $0.1 \%$ Tween-20. Fixed cells were permeabilized in $0.5 \%$ Triton $\mathrm{X}-100$ for $10 \mathrm{~min}$ at room temperature, followed by three washes in PBS with $0.1 \%$ Tween-20. The next step was, blocking with $3 \%$ bovine serum albumin in PBS for $1 \mathrm{~h}$, also at room temperature. After three washes, EMP2 primary antibody or TRP-1 primary antibody or TRP-2 primary antibody or MITF primary antibody were incubated with coverslips overnight at $4^{\circ} \mathrm{C}$. After three washes in PBS with $0.1 \%$ 
Tween 20, cells were treated with species-specific second antibodies conjugated to goat anti-mouse IgG antibody (Alexa Fluor $^{\mathrm{TM}}$ 488, 1:500; Molecular Probes) and goat anti-rabbit IgG antibody (Alexa Fluor ${ }^{\mathrm{TM}}$ 594, 1:500; Molecular Probes). The MNT1 cells were then reacted with the coverslips for $1 \mathrm{~h}$ at room temperature, followed by four washes in PBS with $0.1 \%$ Tween-20. The final samples were mounted onto slides and visualized by confocal microscopy.

\section{Invasion assay}

Invasion assays were performed using transwells that were coated with Matrigel $(0.5 \mu \mathrm{g} / \mathrm{mL}$ ) (Neuro Probe Inc., Gaithersburg, MD) following a previous report (Choi et al., 2020). Coat Matrigel $(10 \mu \mathrm{L} /$ well $)$ in the transwell insert, overnight at room temperature. Trypsinized cells were suspended in a serumfree medium, and $2 \times 10^{6}$ cells were added to the upper chamber of the transwell inserts. Medium with $20 \%$ FBS was added to the lower chamber. After $48 \mathrm{~h}$ incubation with MNT1 cells, the non-invaded cells on the upper surface of the membrane were removed, the invaded cells on the lower surface of the membrane were fixed with cold methanol. And then transwell membranes were stained with hematoxylin and eosin. The cells were photographed (200x magnification) and cells in 3 randomly selected fields were counted. All experiments were repeated at least three times with two replicates each.

\section{Migration assay}

Cell migration was also studied using transwell inserts (Neuro Probe Inc., Gaithersburg, MD, USA) coated with 10 $\mu \mathrm{g} / \mathrm{mL}$ fibronectin as described previously (Lee et al., 2020). Trypsinised cells were suspended in a serum-free medium, and $2 \times 10^{6}$ cells were added to the upper chamber of the transwell inserts. Medium with $3 \%$ FBS was added to the lower chamber incubate for $10 \mathrm{~min}$. After $37^{\circ} \mathrm{C}$ incubation for 24 $\mathrm{h}$, non-migrated cells on the upper surface of the membrane were removed. Transwell membranes were fixed and stained by Diff-quick ${ }^{\circledR}$. The cells were photographed (200× magnification) and cells in 3 randomly selected fields were counted. All experiments were repeated at least three times with two replicates each.

\section{Construction of MNT1 EMP2 CRISPR/Cas9 cell lines}

EMP2 CRISPR/Cas9 KO plasmid and HDR plasmid were purchased from Santa Cruz Biotechnology. For CRISPR/Cas9 $\mathrm{KO}$ experiments, $5 \times 10^{5}$ MNT1 cells seed in 6 well plates in $3 \mathrm{~mL}$ antibiotic-free growth medium per well until they were grown $60 \%$ confluency (Lin et al., 2021). MNT1 cells were cotransfected with CRISPR/Cas9 KO plasmid and EMP2 HDR plasmid according to the manufacturer's instructions. After cells were co-transfected with CRISPR/Cas9 KO plasmid and HDR plasmid, cells were selected with media containing $5 \mu \mathrm{g} /$ $\mathrm{mL}$ puromycin dihydrochloride (Macrogen, Seoul, Korea). And then selected cells were used for Western blot.

\section{Melanosome transfer}

Melanosome transfer was conducted in accordance with a previous report (Kim et al., 2017). MNT1 melanoma cells $3 \times 10^{4}$ were seeded on coverslips on 12 well plates. After $24 \mathrm{~h}, \mathrm{HaCaT}$ (Human keratinocyte cells) cells were added when changing the 1:1 minimum essential medium/RPMI medium. Cells were co-cultured for $48 \mathrm{~h}$. Co-cultures were washed phosphatebuffered saline (PBS) and fixed with $4 \%$ paraformaldehyde for
$10 \mathrm{~min}$ at room temperature. The fixed cells were followed by three washes in phosphate-buffered saline (PBS) with $0.1 \%$ Tween-20. Fixed cells were permeabilized in $0.5 \%$ Triton X-100 for $10 \mathrm{~min}$ at room temperature, followed by three washes in PBS with $0.1 \%$ Tween-20. The next step was, blocking with $3 \%$ bovine serum albumin in PBS for $1 \mathrm{~h}$, also at room temperature. After three washes, EMP2 primary antibody or PMEL primary anti-body were incubated with coverslips overnight at $4^{\circ} \mathrm{C}$. After three washes in PBS with $0.1 \%$ Tween 20 , cells were treated with species-specific second antibodies conjugated to donkey anti-rabbit IgG antibody (Alexa Fluor ${ }^{\mathrm{TM}}$ 488, 1:500; Molecular Probes) and goat anti-rabbit IgG antibody (Alexa Fluor ${ }^{\mathrm{TM}} 594$, 1:500; Molecular Probes). Co-cultured cells were then reacted with the coverslips for $1 \mathrm{~h}$ at room temperature, followed by four washes in PBS with $0.1 \%$ Tween-20. The final samples were mounted onto slides and visualized by confocal microscopy.

\section{Statistical analysis}

Data are presented as mean \pm standard error of the mean (SD) for at least three independent experiments performed in triplicate. Statistical analysis involved the paired Student's ttest. Differences were considered statistically significant at a level of $p<0.05$.

\section{RESULTS}

\section{EMP2 expression is correlated with melanogenic enzyme activity}

In the A375 human melanoma cell line, EMP2 acts as a tumour suppressor by inducing apoptosis (Ahmat Amin et al., 2019). However, the effect of EMP2 on melanogenesis has not yet been investigated. Therefore, we investigated the relationship between EMP2 and the melanogenesis-related genes TYR, TRP-1, and TRP-2 using bioinformatics analysis.

Using the correlation analysis module of GEPIA 2, we investigated the correlation of EMP2 expression with that of TRP-1, TRP-2 and TYR in the GTEx skin dataset (GTEx Consortium, 2017). In sun-exposed skin, EMP2 expression was positively correlated with TYR, TRP-1, and TRP-2 expression ( $R=0.3$, $p=0.0 ; \mathrm{R}=0.23, p=0.0 ; \mathrm{R}=0.24, p=0.0$ ) (Fig. 1A left, 1B left, 1C left). It showed a significant positive correlation value $(R=0.3$, $p=0.0$ ) with TYR (Fig. 1A, left). However, in normal skin not exposed to the sun, the expression of TYR and TRP-1 had a decreased correlation with the expression of EMP2 ( $R=0.22$; $p=0.00056 ; \mathrm{R}=0.17, p=0.00096 ; \mathrm{R}=0.24, p=0.0003$ ) (Fig. $1 \mathrm{~A}$ right, $1 \mathrm{~B}$ right, $1 \mathrm{C}$ right). These results suggest that the expression of EMP2 is correlated to the expression of melanogenesisrelated players such as TYR, TRP-1, and TRP-2.

\section{Loss of EMP2 suppresses melanogenesis and invasion and migration of MNT1 melanoma cells}

To determine whether EMP2 affects melanogenesis, we investigated the effect of EMP2 gene silencing on TYR, TRP-1, and TRP-2 expressions in melanogenesis-prone MNT1 cells. When EMP2 expression was suppressed in MNT1 melanoma cells, TYR and TRP-2 were significantly down-regulated at both the protein and mRNA levels, but the expression of TRP-1 was not affected (Fig. 2A, 2B). We also performed confocal microscopy of EMP2, TRP-1, TRP-2, and MITF in MNT1 melanoma cells. EMP2 loss decreased TRP-2 expression but had little effect on MITF expression (Fig. 2C). 
A
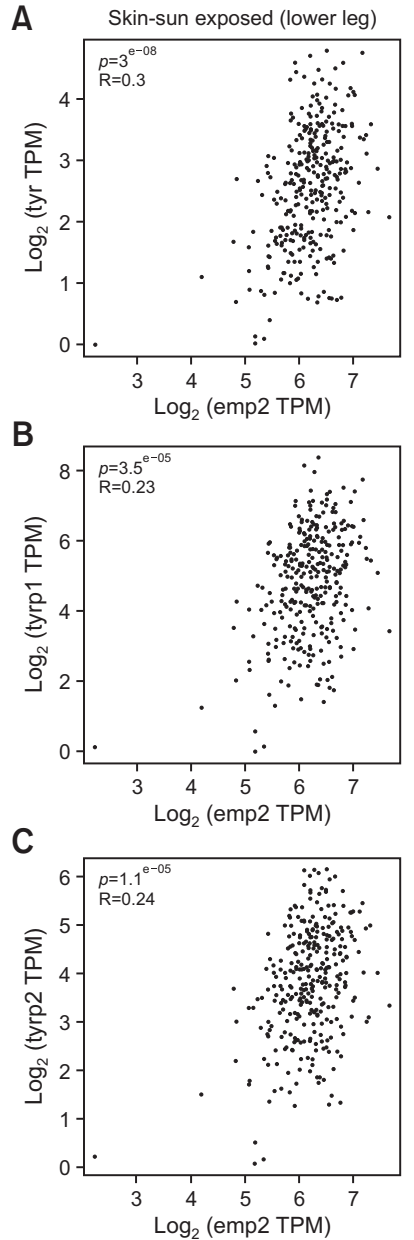

Skin-not sun exposed (suprapubic)
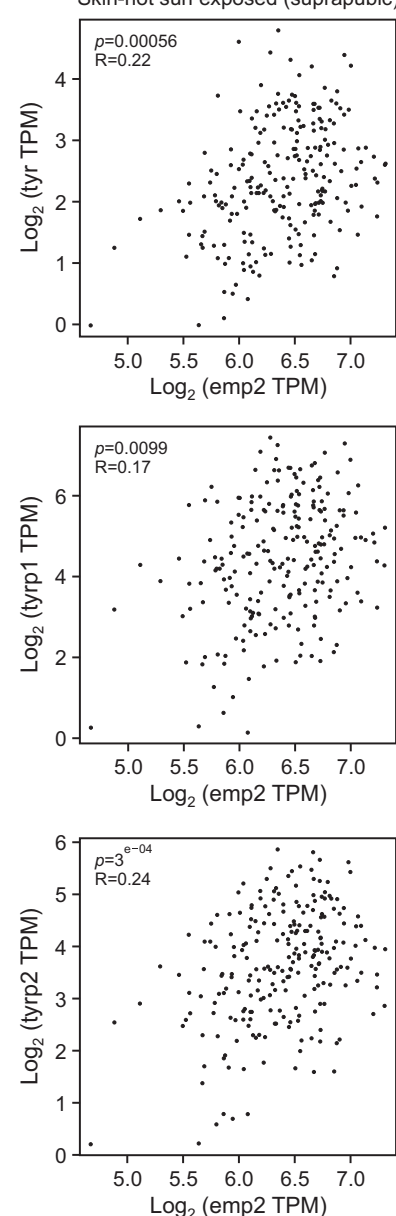

Fig. 1. The relationship of EMP2 expression levels with TRP-1 TRP-2, and TYR expressions in the GTEx skin dataset analyzed in GEPIA. The correlation of EMP2 expression levels with TRP-1, TRP-2 (DCT), and TYR expressions in the GTEx skin dataset was analyzed by Pearson's correlation coefficient. Abbreviations: GEPIA, Gene Expression Profiling Interactive Analysis; GTEx, Genotype-Tissue expression; TPM, transcripts per kilobase million (A), TRP-1 (B), TRP-2 (C), TYR.

The migration of melanocytes to areas where melanocytes are scarce is also an important process in melanogenesis (Jeon et al., 2007; Lee, 2015; Enkhtaivan and Lee, 2021). Therefore, we investigated the effect of EMP2 on cell invasion/migration using the Matrigel-coated assay and the transwell assay. Our results showed that invasion and migration were reduced in MNT1 cells with reduced EMP2 expression by EMP2 siRNA compared with control cells (Fig. 2D, 2E).

\section{Overexpression of EMP2 enhances the melanogenesis, invasion, and migration of MNT1 melanoma cells}

EMP2 overexpression increased the protein and mRNA expression of key enzymes regulating pigment production, such as TRP-2 and TYR, in MNT1 cells but had little effect on TRP-1 expression (Fig. 3A, 3B). We also performed confocal microscopy analysis of EMP2, TRP-1, TRP-2 and MITF in MNT1 melanoma cells. We found that overexpression of EMP2 increased TRP-2 expression but had little effect on

TRP-1 and MITF expressions (Fig. 3C). We then sought to determine whether overexpression of EMP2 affects MNT1 melanoma cell migration and invasion. We determined the effect of EMP2 overexpression on cell invasion/migration using the Matrigel-coated and transwell assays. Our results indicated that invasion and migration of MNT1 cells transfected with the EMP2 plasmid were increased compared to control cells. (Fig. 3D, 3E).

\section{EMP2 KO suppresses melanogenesis and invasion and migration of MNT1 melanoma cells and melanosome transfer of coculture of MNT1 melanoma cells with HaCaT cells}

In recent years, CRISPR gene repair technology has become the most powerful approach to generating genetic models for both basic and preclinical research (Doudna and Charpentier, 2014; Lin et al., 2021). CRISPR/Cas9 is a targeted genome-editing tool. In this study, we used the CRISPR/Cas9 system to create a stable cell line lacking EMP2 to confirm the above results (Fig. 4A, top). It was found that the amount of melanin pigment in the cell line lacking EMP2 was noticeably lower (Fig. 4A, bottom). In the EMP2 knockout cell line, the protein and $m R N A$ expressions of TRP-2 and TYR were significantly decreased compared to the control cells (Fig. 4B, 4C). However, there was no significant effect on TRP-1 expression. We conducted the same cell invasion and migration assays to determine the effect of EMP2 knocking out on cell invasion and migration. Our results indicated that the invasion and migration of MNT1 cells co-transfected with the EMP2 CRISPR/ Cas9 and EMP2 HDR plasmids were lower than those of the control cells (Fig. 4D, 4E).

Melanin pigment is synthesized in melanocytes and accumulates in organelles called melanosomes. These melanosomes travel to the dendrites of the melanocytes and are delivered to nearby KC (Lee, 2015; Enkhtaivan and Lee, 2021). To determine whether the lack of EMP2 affects the transport of $\mathrm{KC}$ in the melanosome, MNT1 cells and HaCaT cells (KC) were cocultured to investigate whether the expression of PMEL, a marker of melanosome, was observed in KC under a confocal microscope. As a result, melanosome transfer from wild-type MNT cells to HaCaT cells was confirmed in control cells, whereas melanosome transfer was significantly reduced in the coculture lacking EMP2 MNT cells (Fig. 4F).

\section{DISCUSSION}

The skin is an important organ that protects against mechanical shock and pressure, temperature changes, microorganisms, radioactive substances, and chemicals (Chuong et al., 2002; Tobin, 2006). While performing these protective functions, skin damage, darkening, excessive pigmentation, and even skin cancer can occur in the skin. Skin colour is determined by a pigment called melanin, which is produced by melanocytes (Costin and Hearing, 2007). However, exposure to UVA/UVB, chemicals, and radioactive substances over-activates melanin production, which can lead to various skin hyperpigmentation diseases, such as melasma and postinflammatory pigmentation (Lee, 2015; Enkhtaivan and Lee, 2021). For the treatment of these skin pigment diseases, research on the process of pigmentation and related genes and enzymes is required continuously. 
A

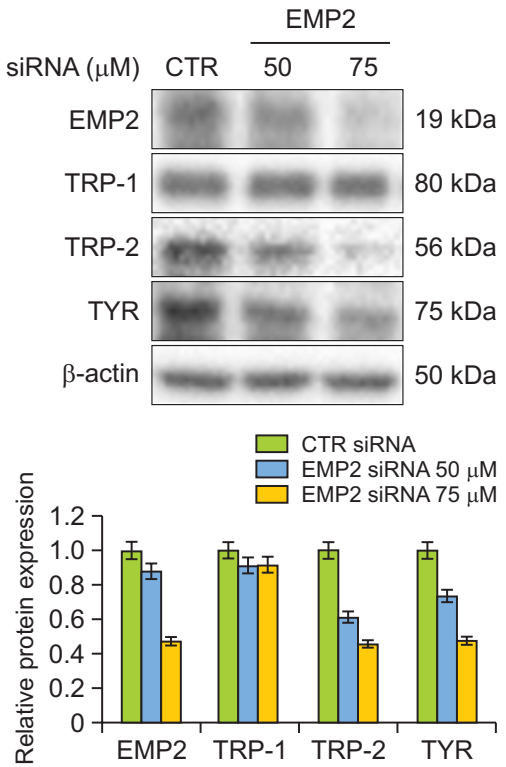

C
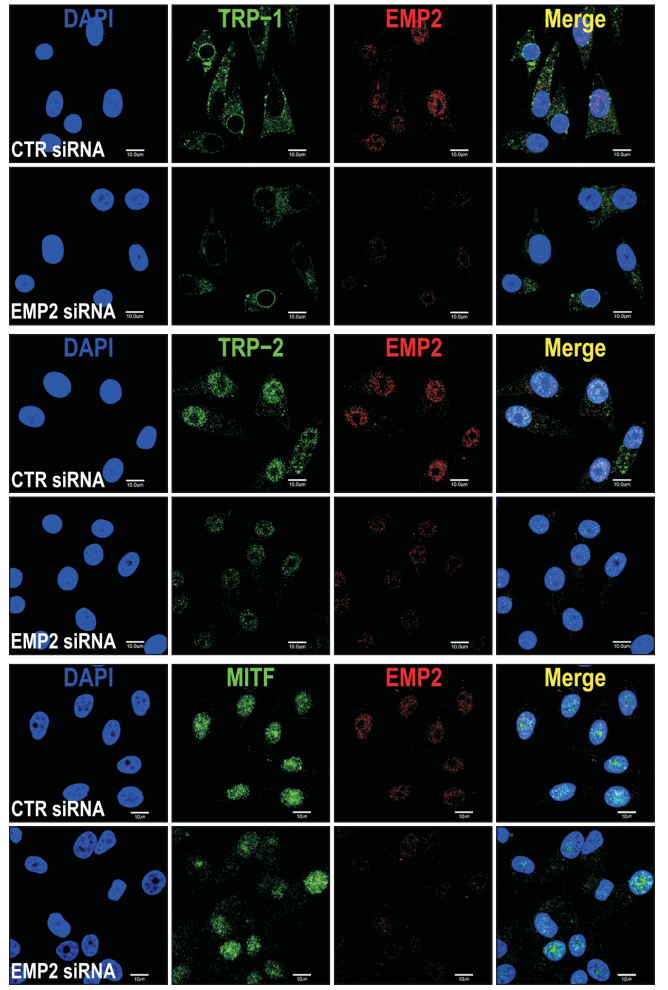

Fig. 2. Loss of EMP2 suppresses TRP-2 and TYR expression levels and invasion and migration of MNT1 melanoma cells. MNT1 cells were transfected with negative control siRNA and EMP2-specific siRNA. (A, top) The protein expressions of EMP2, TRP-1, TRP-2, and TYR were analyzed using Western blotting with $\beta$-actin as a loading control. (A, bottom) Densitometric analysis of EMP2, TRP-1, TRP-2, and TYR from Western blots. (B, top) EMP2, TRP-1, TRP-2, and TYR mRNA transcript levels were analyzed by RT-PCR, with GAPDH as a loading control. (B, bottom) Densitometric analysis of EMP2, TRP-1, TRP-2, and TYR from RT-PCR. (C) The localization of EMP2, TRP-1, TRP-2, and MITF. Immunostaining was performed using MITF, TRP-1, TRP-2 (green), EMP2 (red), and DAPI (blue). (D) MNT1 melanoma cells were transfected with EMP2-specific siRNA for $48 \mathrm{~h}$, and lower chamber transwells were coated with Matrigel $(0.5 \mu \mathrm{g} / \mathrm{mL})$. (D, top) Loss of EMP2 reduced MNT1 melanoma cell invasion. (D, bottom) Images of invaded MNT1 melanoma cells. (E) MNT1 melanoma cells were transfected with EMP2-specific siRNA for $48 \mathrm{~h}$ and allowed to migrate through fibronectin $(10 \mu \mathrm{g} / \mathrm{mL})$-coated membrane in transwells. (E, top) Loss of EMP2 reduced MNT1 melanoma cell migration. (E, bottom) Images of migrated MNT1 melanoma cells. A * $p$ value $<0.05$ was considered significant and error bars, \pm SD. 
A
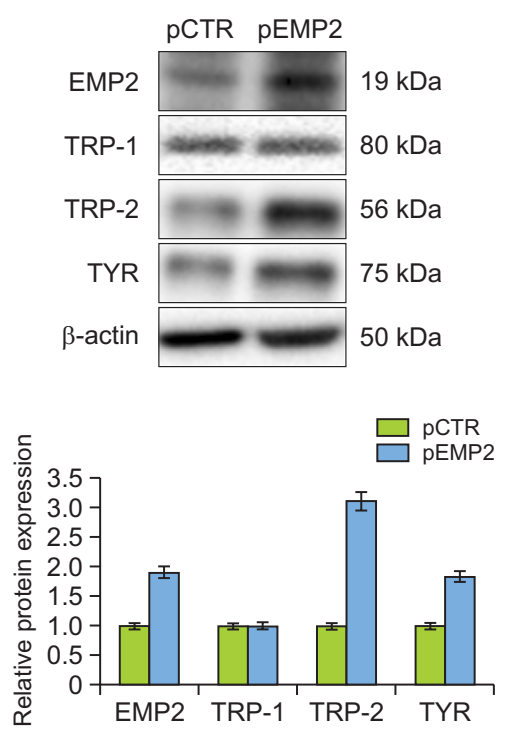

C
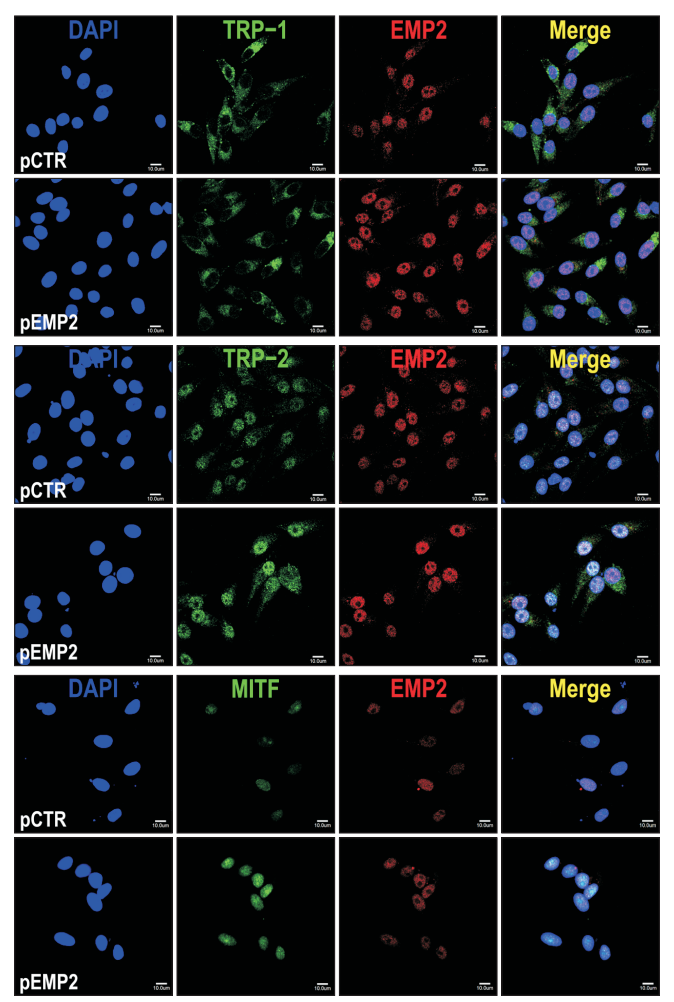

B
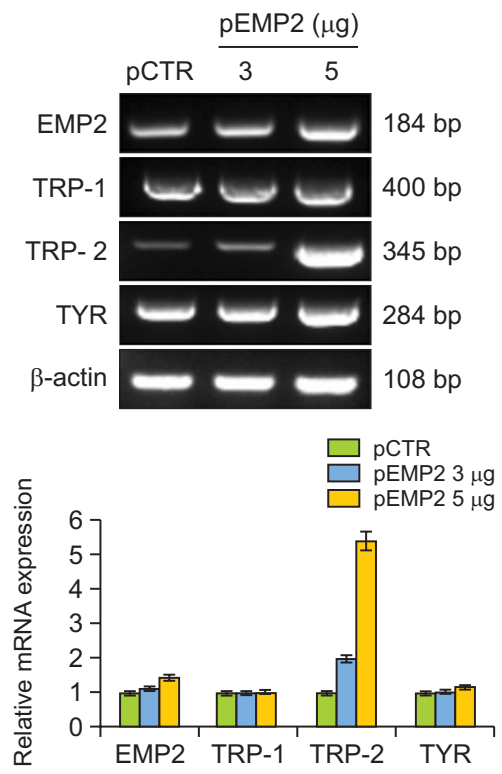

D
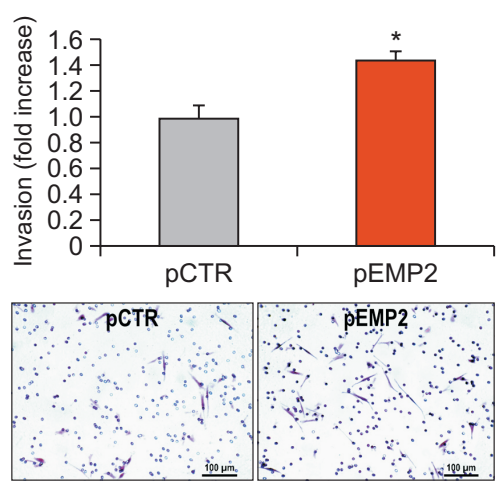

E
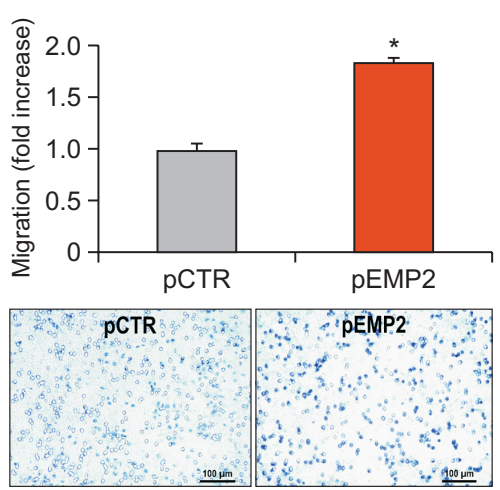

Fig. 3. Overexpression of EMP2 enhances TRP-2 and TYR expression levels and invasion and migration of MNT1 melanoma cells. MNT1 cells were transfected with a plasmid containing EMP2 and control plasmid DNA. (A, top) EMP2, TRP-1, TRP-2, and TYR protein expression levels were analyzed using Western blotting, with $\beta$-actin as a loading control. (A, bottom) Densitometric analysis of EMP2, TRP-1, TRP-2, and TYR from Western blots. (B, top) EMP2, TRP-1, TRP-2, and TYR mRNA levels were analyzed using RT-PCR, with GAPDH as a loading control. (B, bottom) Densitometric analysis of EMP2, TRP-1, TRP-2, and TYR from RT-PCR. (C) The localization of EMP2, TRP-1, TRP-2, and MITF. Immunostaining was performed using MITF, TRP-1, TRP-2 (green), EMP2 (red), and DAPI (blue). (D) MNT1 melanoma cells were transfected with EMP2 plasmid DNA for $48 \mathrm{~h}$, and lower chamber transwells were coated with Matrigel $(0.5 \mu \mathrm{g} / \mathrm{mL})$. (D, top) Overexpressed EMP2 enhanced MNT1 melanoma cell invasion. (D, bottom) Images of invaded MNT1 melanoma cells. (E) MNT1 melanoma cells were transfected with EMP2 plasmid DNA for $48 \mathrm{~h}$ and allowed to migrate through fibronectin $(10 \mu \mathrm{g} / \mathrm{mL})$-coated membrane in transwells. (E, top) Overexpressed EMP2 enhanced MNT1 melanoma cell migration. (E, bottom) Images of migrated MNT1 melanoma cells. $A^{*} p$ value $<0.05$ was considered significant and error bars, \pm SD. 
A

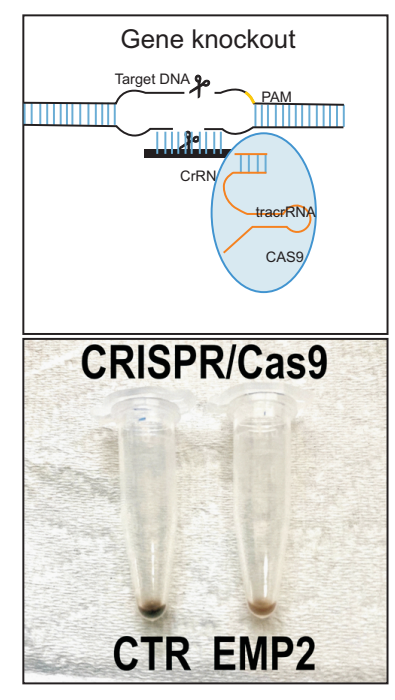

D
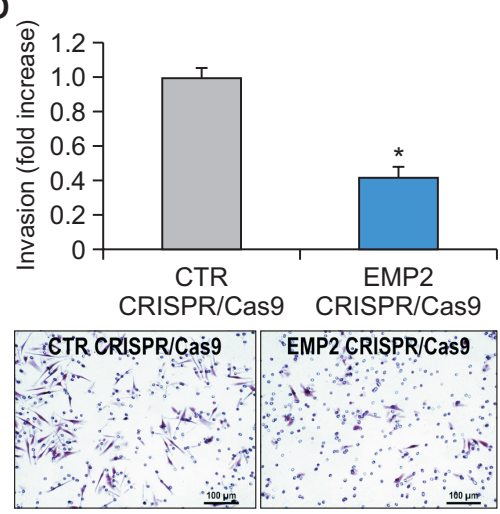

F
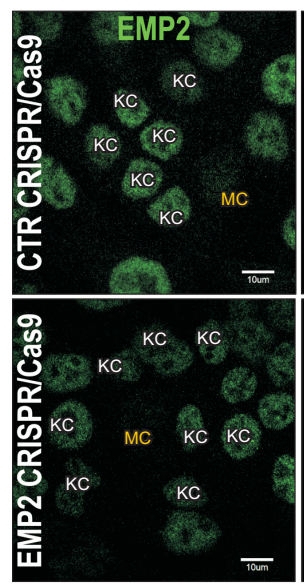

B

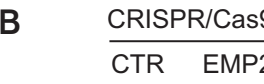

EMP2

TRP-1

TRP-2

TYR

$\beta$-actin

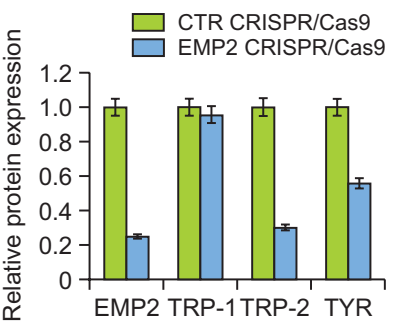

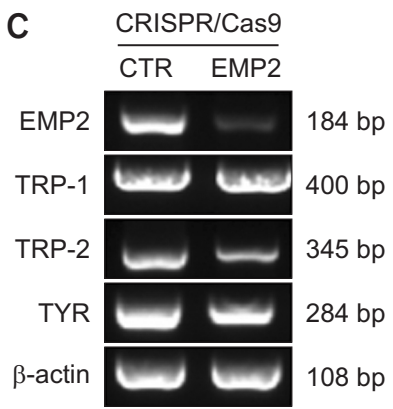

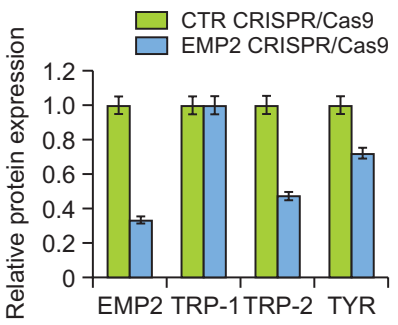

E
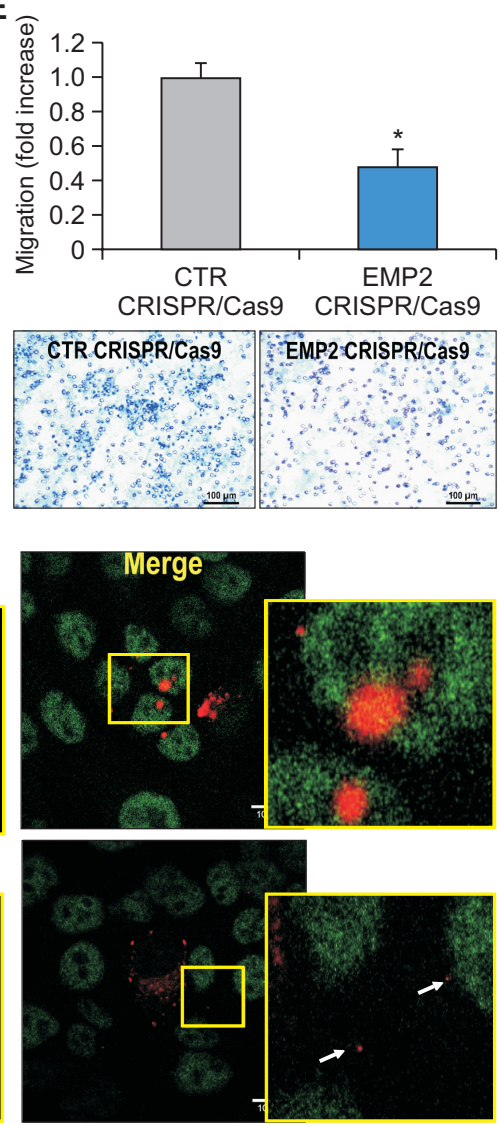

Fig. 4. EMP2 KO decreases expressions of TRP-2 and TYR in MNT1 melanoma cells and melanosome transfer in coculture of MNT1 and HaCaT cells. (A top) Scheme of EMP2 KO by CRISPR/Cas9. (A, bottom) MNT1 cells were co-transfected with the control CRISPR/Cas9 plasmid and EMP2 CRISPR/Cas9 plasmid/HDR plasmid. Images of the effect of EMP2 gene KO in MNT1 cells. (B, top) EMP2, TRP-1, TRP-2, and TYR protein expressions were analyzed using Western blotting, with $\beta$-actin as a loading control. (B, bottom) Densitometric analysis of EMP2, TRP-1, TRP-2, and TYR from Western blots. (C, top) EMP2, TRP-1, TRP-2, and TYR mRNA expression levels were analyzed using RT-PCR, with GAPDH as a loading control. (C, bottom) Densitometric analysis of EMP2, TRP-1, TRP-2, and TYR from RT-PCR. (D, E) EMP2 KO reduces the invasion and migration of MNT1 melanoma cells. (D) MNT1 melanoma cells were co-transfected with EMP2 CRISPR/Cas9 plasmid and HDR plasmid, and lower chamber transwells were coated with Matrigel $(0.5 \mu \mathrm{g} / \mathrm{mL})$. (D, top) EMP2 KO reduced MNT1 melanoma cell invasion. (D, bottom) Images of invaded MNT1 melanoma cells. (E) MNT1 melanoma cells were co-transfected with specific CRISPR/Cas9 plasmid and HDR plasmid targeting EMP2 and allowed to migrate through fibronectin $(10 \mu \mathrm{g} / \mathrm{mL})$-coated membrane in transwells. (E, top) EMP2 KO reduced MNT1 melanoma cell migration. (E, bottom) Images of migrated MNT1 melanoma cells. (F) Confocal microscopic examination of PMEL and EMP2 in MNT1/HaCaT coculture cells. Double immunolabeling of MC/KC cocultures with PMEL antibody (red) and anti-EMP2 antibody (green). KC: keratinocyte, MC: melanocyte, PMEL: melanosome marker. ${ }^{*} p$ value $<0.05$ was considered significant and error bars, \pm SD. 
EMP2 is a cell membrane protein and is known as a cancer biomarker and therapeutic target. It functions as an oncogene in brain cancer and endometrial cancer and as a tumour suppressor gene in B cell lymphoma, lung cancer, melanoma, and bladder cancer (Wang et al., 2001; Qin et al., 2014; Li et al., 2015; Lee et al., 2016; Kiyohara et al., 2017; Wang et al., 2019; Li et al., 2021; Ma et al., 2021). EMP2 has been identified as a novel melanoma suppressor regulated by mTORmediated autophagy (Wang et al., 2019). However, there are no detailed studies of the link between EMP2 and skin pigmentation. Using the GEPIA2 tool (Fig. 1), we found that EMP2 is associated with two melanogenesis-related enzymes TYR and TRP-2. Therefore, we conducted a study on the role of EMP2 in melanogenesis.

Our results revealed a role for EMP2 in the pigmentation of MNT1 melanoma cells. EMP2 deficiency reduced the expression of the major melanin biosynthetic enzymes TRP-2 and TYR (Fig. 2, 4). Conversely, overexpression of EMP2 increased the expression levels of TYR and TRP-2 (Fig. 3), and this effect was most remarkable for TRP-2. This strong effect of EMP2 on TRP-2 expression may be associated with the fact that TRP-2 and TRP-1 are trafficked by distinct pathways in B16 melanoma cells (Negroiu et al., 2005). Thus, it seems highly likely that EMP2 is involved in an early secretory pathway that includes TRP-2 as cargo but not TRP-1. However, more research is required to confirm this proposition (Negroiu et al., 2005). Interestingly, EMP2, present in the perinuclear region, is also known to be present in the Golgi region (Wadehra et al., 2004). As a result of confirming the locations and expressions of EMP2, TRP-1, TRP-2, and MITF using confocal microscopy, it was found that the expression of TRP-2 was lower (Fig. 3E). These data confirm that loss of EMP2 reduces melanogenesis, but more research is needed to decipher the mechanisms.

EMP2 depletion decreased MNT1 cell invasion and migration, and increased EMP2 expression increased cell invasion and migration (Fig. 2D, 2E, 3D, 3E, 4D, 4E). The report that the loss of EMP2 reduces cell migration is consistent with the inhibition of neutrophil transendothelial migration in EMP2-/mice (Lin et al., 2020). Moreover, an increase in EMP2 expression is consistent with previous findings that it also increases the migration of glioblastoma cells. However, the results conflict with studies that loss of EMP2 in lung cancer cell lines increases cell migration and invasion (Lee et al., 2016; Ma et al., 2021). This difference seems to be related to the cell context or the microenvironment surrounding the cells.

Melasma is hypermelanosis required for sun-exposed skin and is increased by $50-70 \%$ of pregnant women and menstruation (Bolanca et al., 2008; Lee, 2015; Mobasher et al., 2020). Estrogens, particularly sex hormones, such as $17 \beta$-estradiol (E2) and progesterone, are factors involved in the regulation of pigmentation (Natale et al., 2016). Estradiol levels are higher in the serum of women with melasma than in control women without melasma (Hassan et al., 1998). Interestingly, EMP2 expression is increased by estrogen and progesterone (Wadehra et al., 2008). Therefore, the involvement of EMP2 in melanogenesis may partially explain the increase in freckles caused by hormones, such as estrogen, during pregnancy. Conversely, if EMP2 expression is increased, it seems that symptoms can be improved by increasing melanogenesis in skin diseases caused by pigment deficiency, such as vitiligo. In conclusion, EMP2 represents a potential therapeutic target for the treatment of various skin pigmentation diseases.

\section{CONFLICT OF INTEREST}

The authors have declared that no competing interest exists.

\section{ACKNOWLEDGMENTS}

This study was supported by a grant from the Basic Science Research Program through the NRF (NRF2018R1A5A2023127, NRF-2020R1A2C3004973, and NRF2020M3E5E20383 56), the Korea Health Technology R\&D Project through the Korea Health Industry Development Institute (KHIDI), funded by the Ministry of Health \& Welfare, Korea (HP20C0131), and the BK21 FOUR program through the National Research Foundation (NRF) of Korea funded by the Ministry of Education (MOE, Korea).

\section{REFERENCES}

Ahmat Amin, M. K. B., Shimizu, A. and Ogita, H. (2019) The pivotal roles of the epithelial membrane protein family in cancer invasiveness and metastasis. Cancers 11, 1620.

Bolanca, I., Bolanca, Z., Kuna, K., Vukovic, A., Tuckar, N., Herman, R. and Grubisic, G. (2008) Chloasma--the mask of pregnancy. Coll. Antropol. 32 Suppl 2, 139-141.

Choi, E. K., Park, E. J., Phan, T. T., Kim, H. D., Hoe, K. L. and Kim, D. U. (2020) Econazole induces P53-dependent apoptosis and decreases metastasis ability in gastric cancer cells. Biomol. Ther. (Seoul) 28, 370-379.

Chuong, C. M., Nickoloff, B., Elias, P., Goldsmith, L., Macher, E., Maderson, P., Sundberg, J., Tagami, H., Plonka, P., ThestrupPederson, K., Bernard, B. A., Schröder, J. M., Dotto, P., Chang, C. M., Williams, M. L., Feingold, K. R., King, L. E., Kligman, A. M., Rees, J. L. and Christophers, E. (2002) What is the 'true' function of skin? Exp. Dermatol. 11, 159-187.

Costin, G. E. and Hearing, V. J. (2007) Human skin pigmentation: melanocytes modulate skin color in response to stress. FASEB J. 21, 976-994.

Doudna, J. A. and Charpentier, E. (2014) The new frontier of genome engineering with CRISPR-Cas9. Science 346, 1258096.

Enkhtaivan, E. and Lee, C. H. (2021) Role of amine neurotransmitters and their receptors in skin pigmentation: therapeutic implication. Int. J. Mol. Sci. 22, 8071.

GTEx Consortium (2017) Genetic effects on gene expression across human tissues. Nature 550, 204-213.

Hassan, I., Kaur, I., Sialy, R. and Dash, R. J. (1998) Hormonal milieu in the maintenance of melasma in fertile women. J. Dermatol. 25, 510-512.

Ito, Y. and Sato, K. (2021) Salicylamide enhances melanin synthesis in B16F1 melanoma cells. Biomol. Ther. (Seoul) 21, 445-451.

Jeon, S., Kim, N. H., Koo, B. S., Lee, H. J. and Lee, A. Y. (2007) Bee venom stimulates human melanocyte proliferation, melanogenesis, dendricity and migration. Exp. Mol. Med. 39, 603-613.

Kim, H. J., Kim, B., Byun, H. J., Yu, L., Nguyen, T. M., Nguyen, T. H., Do, P. A., Kim, E. J., Cheong, K. A., Kim, K. S., Huy Phùng, H., Rahman, M., Jang, J. Y., Rho, S. B., Kang, G. J., Park, M. K., Lee, H., Lee, K., Cho, J., Han, H. K., Kim, S. G., Lee, A. Y. and Lee, C. H. (2021a) Resolvin D1 suppresses H2O2-induced senescence in fibroblasts by inducing autophagy through the miR-1299/ARG2/ ARL1 axis. Antioxidants 10, 1924.

Kim, H. J., Park, M. K., Byun, H. J., Kim, M., Kim, B., Yu, L., Nguyen, T. M., Nguyen, T. H., Do, P. A., Kim, E. J., Kim, J. H., Enkhtaivan, E., Kim K. S., Jang, J. Y., Kang, G. J., Lee, H., Won M., Lee, K., Cho, 
J. and Lee, C. H. (2021b) LW1497, an inhibitor of malate dehydrogenase, suppresses TGF- $\beta 1$-induced epithelial-mesenchymal transition in lung cancer cells by downregulating slug. Antioxidants 10, 1674.

Kim, H. J., Lee, H. J., Park, M. K., Gang, K. J., Byun, H. J., Park, J. H., Kim, M. K., Kim, S. Y. and Lee, C. H. (2014) Involvement of transglutaminase-2 in $\alpha-\mathrm{MSH}$-induced melanogenesis in SK-MEL-2 human melanoma cells. Biomol. Ther. (Seoul) 22, 207-212.

Kim, N. H., Choi, S. H., Yi, N., Lee, T. R. and Lee, A. Y. (2017) Arginase-2, a miR-1299 target, enhances pigmentation in melasma by reducing melanosome degradation via senescence-induced autophagy inhibition. Pigment Cell Melanoma Res. 30, 521-530.

Kiyohara, M. H., Dillard, C., Tsui, J., Kim, S. R., Lu, J., Sachdev, D. Goodglick, L., Tong, M., Torous, V. F., Aryasomayajula, C., Wang, W., Najafzadeh, P., Gordon, L. K., Braun, J., McDermott, S., Wicha, M. S. and Wadehra, M. (2017) EMP2 is a novel therapeutic target for endometrial cancer stem cells. Oncogene 36, 5793-5807.

Kobayashi, T., Urabe, K., Winder, A., Jiménez-Cervantes, C., Imokawa, G., Brewington, T., Solano, F., García-Borrón, J. and Hearing, V. (1994) Tyrosinase related protein 1 (TRP1) functions as a DHICA oxidase in melanin biosynthesis. EMBO J. 13, 5818-5825.

Lambert, M. W., Maddukuri, S., Karanfilian, K. M., Elias, M. L. and Lambert, W. C. (2019) The physiology of melanin deposition in health and disease. Clin. Dermatol. 37, 402-417.

Lee, A. Y. (2015) Recent progress in melasma pathogenesis. Pigment Cell Melanoma Res. 28, 648-660.

Lee, H., Park, M., Kim, S., Park Choo, H., Lee, A. and Lee, C. (2011) Serotonin induces melanogenesis via serotonin receptor $2 A$. BR. J. Dermatol. 165, 1344-1348.

Lee, J., Yoon, S. S., Thuy, P. X. and Moon, E. Y. (2020) Synovial cell migration is associated with $B$ cell activating factor expression increased by TNF $\alpha$ or decreased by KR33426. Biomol. Ther. (Seoul) 28, 405-413.

Lee, E. J., Park, M. K., Kim, H. J., Kim, E. J., Kang, G. J., Byun, H. J. and Lee, C. H. (2016) Epithelial membrane protein 2 regulates sphingosylphosphorylcholine-induced keratin 8 phosphorylation and reorganization: changes of PP2A expression by interaction with alpha4 and caveolin-1 in lung cancer cells. Biochim. Biophys. Acta Mol. Cell Res. 1863, 1157-1169.

Li, C. F., Chan, T. C., Pan, C. T., Vejvisithsakul, P. P., Lai, J. C., Chen, S. Y., Hsu, Y. W., Shiao, M. S. and Shiue, Y. L. (2021) EMP2 induces cytostasis and apoptosis via the TGF $\beta / S M A D / S P 1$ axis and recruitment of P2RX7 in urinary bladder urothelial carcinoma. Cell. Oncol. 44, 1133-1150.

Li, C. F., Wu, W. J., Wu, W. R., Liao, Y. J., Chen, L. R., Huang, C. N., Li, C. C., Li, W. M., Huang, H. Y., Chen, Y. L., Liang, S. S., Chow, N H. and Shiue, Y. L. (2015) The cAMP responsive element binding protein 1 transactivates epithelial membrane protein 2 , a potential tumor suppressor in the urinary bladder urothelial carcinoma. Oncotarget 6, 9220-9239.

Lin, C. L., Tsai, M. L., Chen, Y. h., Liu, W. N., Lin, C. Y., Hsu, K. W., Huang, C. Y., Chang, Y. J., Wei, P. L., Chen, S. H., Huang, L. C. and Lee, C. H. (2021) Platelet-derived growth factor receptor- $\alpha$ subunit targeting suppresses metastasis in advanced thyroid cancer in vitro and in vivo. Biomol. Ther. (Seoul) 29, 551-561.

Lin, W. C., Gowdy, K. M., Madenspacher, J. H., Zemans, R. L., Yamamoto, K., Lyons-Cohen, M., Nakano, H., Janardhan, K., Williams, C. J., Cook, D. N., Mizgerd, J. P. and Fessler, M. B. (2020) Epithelial membrane protein 2 governs transepithelial migration of neutrophils into the airspace. J. Clin. Invest. 130, 157-170.

Liu, Y., Dakou, E., Meng, Y. and Leyns, L. (2019) Loss of Emp2 compromises cardiogenic differentiation in mouse embryonic stem cells. Biochem. Biophys. Res. Commun. 511, 173-178.

Ma, Y., Schröder, D. C., Nenkov, M., Rizwan, M. N., Abubrig, M.,
Sonnemann, J., Murrieta-Coxca, J. M., Morales-Prieto, D. M., Westermann, M., Gaßler, N. and Chen, Y. (2021) Epithelial membrane protein 2 suppresses non-small cell lung cancer cell growth by inhibition of MAPK pathway. Int. J. Mol. Sci. 22, 2944.

Mobasher, P., Foulad, D. P., Raffi, J., Zachary, C., Fackler, N., Zohuri, N., Juhasz, M. and Atanaskova Mesinkovska, N. (2020) Catamenial hyperpigmentation: a review. J. Clin. Aesthet. Dermatol. 13, 18-21.

Natale, C. A., Duperret, E. K., Zhang, J., Sadeghi, R., Dahal, A., O'Brien, K. T., Cookson, R., Winkler, J. D. and Ridky, T. W. (2016) Sex steroids regulate skin pigmentation through nonclassical membrane-bound receptors. Elife 5, e15104.

Negroiu, G., Dwek, R. A. and Petrescu, S. M. (2005) Tyrosinase-related protein-2 and -1 are trafficked on distinct routes in B16 melanoma cells. Biochem. Biophys. Res. Commun. 328, 914-921.

Qin, Y., Fu, M., Takahashi, M., Iwanami, A., Kuga, D., Rao, R. G., Sudhakar, D., Huang, T., Kiyohara, M., Torres, K., Dillard, C., Inagaki, A., Kasahara, N., Goodglick, L., Braun, J., Mischel, P. S., Gordon, L. K. and Wadehra, M. (2014) Epithelial membrane protein-2 (EMP2) activates Src protein and is a novel therapeutic target for glioblastoma. J. Biol. Chem. 289, 13974-13985.

Rho, S. B., Byun, H. J., Kim, B. R. and Lee, C. H. (2021) Knockdown of LKB1 sensitizes endometrial cancer cells via AMPK activation. Biomol. Ther. (Seoul) 29, 650-657.

Serre, C., Busuttil, V. and Botto, J. M. (2018) Intrinsic and extrinsic regulation of human skin melanogenesis and pigmentation. Int. J. Cosmet. Sci. 40, 328-347.

Tang, Z., Kang, B., Li, C., Chen, T. and Zhang, Z. (2019) GEPIA2: an enhanced web server for large-scale expression profiling and interactive analysis. Nucleic Acids Res. 47, W556-W560.

Tobin, D. J. (2006) Biochemistry of human skin-our brain on the outside. Chem. Soc. Rev. 35, 52-67.

Tuerxuntayi, A., Liu, Y.-q., Tulake, A., Kabas, M., Eblimit, A. and Aisa, H. A. (2014) Kaliziri extract upregulates tyrosinase, TRP-1, TRP-2 and MITF expression in murine B16 melanoma cells. BMC Complement. Altern. Med. 14, 166.

Wadehra, M., Goodglick, L. and Braun, J. (2004) The tetraspan protein EMP2 modulates the surface expression of caveolins and glycosylphosphatidyl inositol-linked proteins. Mol. Biol. Cell 15, 20732083.

Wadehra, M., Iyer, R., Goodglick, L. and Braun, J. (2002) The tetraspan protein epithelial membrane protein- 2 interacts with $\beta 1$ integrins and regulates adhesion. J. Biol. Chem. 277, 41094-41100.

Wadehra, M., Mainigi, M., Morales, S. A., Rao, R. G., Gordon, L. K., Williams, C. J. and Braun, J. (2008) Steroid hormone regulation of EMP2 expression and localization in the endometrium. Reprod. Biol. Endocrinol. 6, 15.

Wang, C. X., Wadehra, M., Fisk, B. C., Goodglick, L. and Braun, J. (2001) Epithelial membrane protein 2, a 4-transmembrane protein that suppresses B-cell lymphoma tumorigenicity. Blood 97, 38903895.

Wang, M., Li, S., Zhang, P., Wang, Y., Wang, C., Bai, D. and Jiang, X. (2019) EMP2 acts as a suppressor of melanoma and is negatively regulated by mTOR-mediated autophagy. J. Cancer 10, 35823592.

Wang, Y. W., Cheng, H. L., Ding, Y. R., Chou, L. H. and Chow, N. H. (2017) EMP1, EMP 2, and EMP3 as novel therapeutic targets in human cancer. Biochim. Biophys. Acta Rev. Cancer 1868, 199211.

Yokoyama, K., Yasumoto, K.-i., Suzuki, H. and Shibahara, S. (1994) Cloning of the human DOPAchrome tautomerase/tyrosinase-related protein 2 gene and identification of two regulatory regions required for its pigment cell-specific expression. J. Biol. Chem. 269, 27080-27087. 